\title{
Aneurisma de la Aorta abdominal roto
}

\section{Reporte de un caso}

Camacho-González J*, Martínez-Muñoz F**, Medina-Aguiñaga D**, Sánchez-López J**, Valdivia-Esquivel $R^{* *}$, Flores-Alvarez $E^{* * *}$

\section{Resumen}

La ruptura de un aneurisma aórtico puede cursar con abdomen agudo y presentar datos francos de choque hipovolémico. El propósito del trabajo es reportar el caso de un paciente con ruptura de un aneurisma de la arteria aorta abdominal. Se trata de un hombre de 77 años de edad, portador conocido de un aneurisma aórtico abdominal. El evento agudo ocurrió al momento de levantar un objeto pesado, presentó súbitamente dolor abdominal de gran intensidad y fue llevado al servicio de urgencias donde se le encontró en estado de choque y con un cuadro de abdomen agudo. Se realizo laparotomía de urgencia encontrando ruptura del aneurisma abdominal, se resecó y reparó con prótesis aorto-iliaca. La atención inmediata y el manejo adecuado permitió una recuperación íntegra del paciente.

palabras clave: aneurisma abdominal, choque hipovolémico

\section{Introducción}

El aneurisma de la aorta abdominal (AAA) se define como el aumento del diámetro de la aorta en más del $50 \%$ de su tamaño original. Afecta de manera característica a varones a partir de la séptima década de la vida. Su prevalencia aumenta en nuestro medio paralelamente al incremento de la esperanza de vida y la disminución de la mortalidad cardiovascular. Su rotura es la mayor consecuencia clínica. La rotura aneurismática tiene una mortalidad global cercana al $90 \%{ }^{1}$.

* Angiólogo y Cirujano Vascular, HGZ 1 IMSS, Ags.

** Estudiante de Médicina, UAA.

*** Cirujano Oncólogo, Maestro en Ciencias, HGZ 1 IMSS, Ags. 
La enfermedad aneurismática afecta principalmente a varones de edad avanzada y fumadores de raza blanca. En estudios poblacionales, la prevalencia de aneurismas varía de $1.3 \%$ en varones de 45 a 54 años a $12.5 \%$ en los de 75 a 84 años, con una relación varón:mujer de 4:1. La rotura es una causa frecuente de mortalidad en la población general, con 15,000 muertes por año en Estados Unidos, y constituye la decimoquinta causa de muerte y la décima en varones mayores de 55 años $^{2}$.

La rotura aneurismática es la forma de presentación más grave y supone un compromiso vital si no se trata quirúrgicamente. Ocurre con frecuencia en pacientes con AAA no conocido, por lo que la sospecha clínica es la clave para evitar el retraso en la atención quirúrgica definitiva, uno de los factores relacionados con su alta mortalidad operatoria. ${ }^{3}$ La rotura puede manifestarse clínicamente como contenida en un $2-3 \%$ de los casos, con dolor lumbar, estabilidad hemodinámica y sin hemorragia perivascular. Sin embargo, la forma más frecuente de presentación de la rotura es una hemorragia retroperitoneal $(90 \%)$ con colapso circulatorio, que puede ser transitorio, y un aumento de perímetro abdominal. En ocasiones se manifiesta con sangrado hacia un órgano contiguo, como hacia el intestino ( $1 \%$ ), en el que puede haber sangrado digestivo autolimitado con episodios febriles por bacteriemia, hacia el territorio de la vena iliocava (1\%), con hipertensión venosa, insuficiencia cardiaca, embolismo pulmonar, edema de miembros inferiores o síndrome nefrótico, o como rotura intraperitoneal $(5 \%)$, de consecuencias fatales si no se realiza un tratamiento inmediato. ${ }^{4}$

\section{Presentación del caso}

Paciente masculino de 77 años de edad, con antecedentes de diabetes mellitus tipo 2 e hipertensión arterial sistémica, cáncer broncogénico en tratamiento con radioterapia y se conoce portador de aneurisma de la aorta abdominal. Inició de forma súbita dos horas antes de ingresar al servicio de urgencias del Hospital General de Zona 1 del IMSS, Aguascalientes, con dolor abdominal agudo, continuo, de gran intensidad (Escala Visual Análoga 8/10), palidez de tegumentos y pérdida del estado de alerta después de realizar un esfuerzo físico al levantar un objeto pesado. A la exploración física se le encontró con datos de choque, una presión arterial de $60 / 40 \mathrm{mmHg}$, diaforesis y palidez generalizada de tegumentos, y datos de abdomen agudo. Después de la resucitación inicial con líquidos coloides y cristaloides fue llevado a quirófano donde se realizó una laparotomía exploradora por parte del servicio de Angiología y Cirugía Vascular, se encontró un aneurisma aórtico infrarrenal roto de aproximadamente $15 \times 7 \mathrm{~cm}$., con presencia de un hematoma retroperitoneal difuso de aproxi- 
madamente 1,500 ml. Se procedió a la resección del aneurisma e interposición de una prótesis aortica bifurcada de Dacrón con anastomosis termino-terminal en aorta infrarrenal y termino-lateral con ambas iliacas primitivas. El posoperatorio inmediato fue manejado en la Unidad de Cuidados Intensivos (UCI) con evolución satisfactoria, cursó con estabilidad hemodinámica, sin evidencia de fuga de los sitios de anastomosis ni disfunción respiratoria, renal, hematológica, neurológica ni gastrointestinal. Fue egresado al cuarto día de la $\mathrm{UCl}$ y al séptimo día del hospital en buenas condiciones generales. A tres meses de la cirugía, el paciente se encuentra asintomático y sin datos clínicos ni radiológicos de complicaciones posoperatorias.

Los estudios de imagen realizados antes del cuadro agudo muestran la presencia de un aneurisma aórtico fusiforme, parcialmente trombosado con placas de ateroma calcificadas en sus paredes que compromete la aorta abdominal distal, nace por debajo de la emergencia de las arterias renales y se extiende hasta la región de la bifurcación aorto-iliaca, dando la impresión de comprometer la porción proximal de la arteria iliaca común derecha.

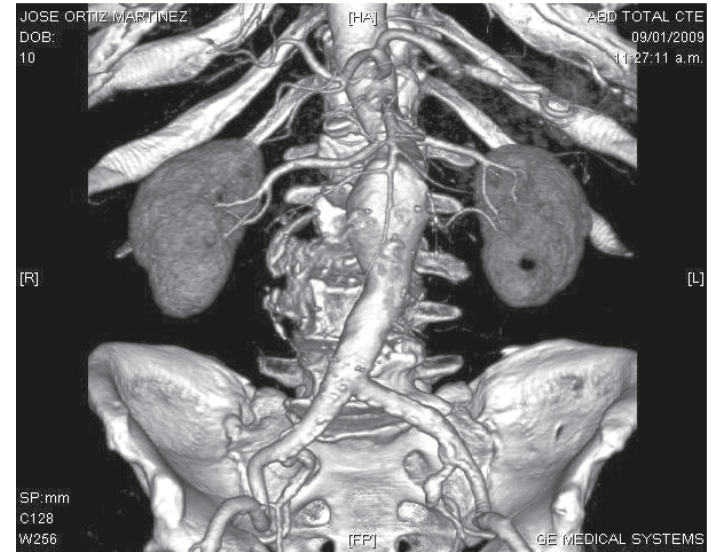

Figura 1. Angio-tomografía que muestra el aneurisma aórtico abdominal (AAA) de localización infrarrenal.

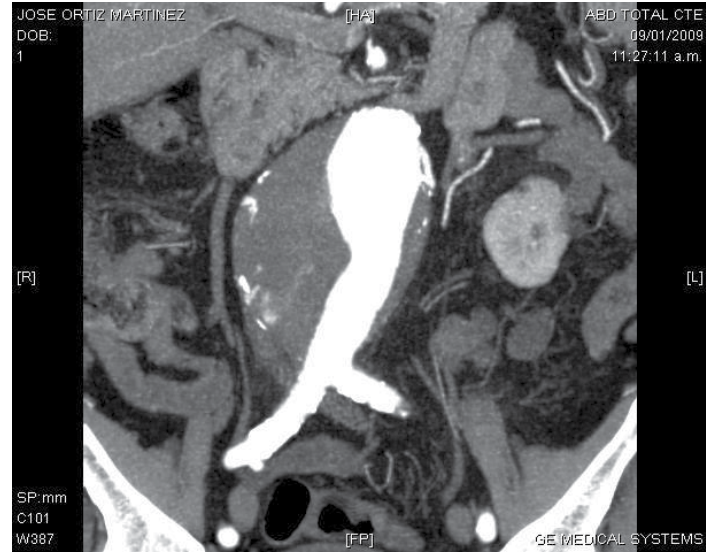

Figura 2. Tomografía contrastada de abdomen, corte coronal que muestra el AAA con la presencia de trombo mural.

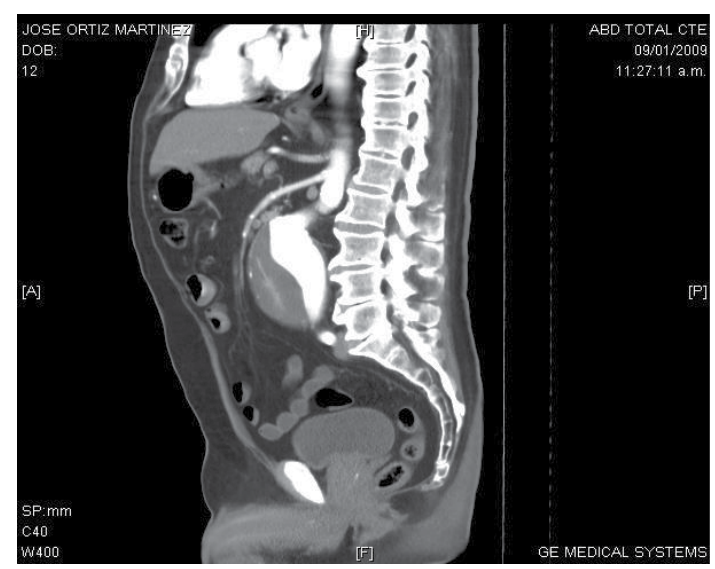

Figura 3. Tomografía contrastada de abdomen, corte sagital que muestra el AAA con la presencia de trombo mural. 


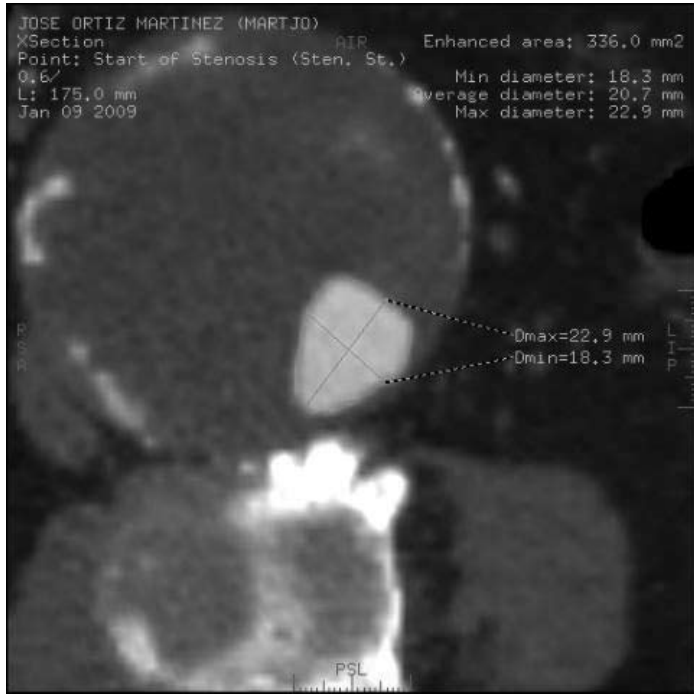

Figura 4. Tomografía contrastada de abdomen, corte transversal que muestra las dimensiones de la luz aórtica dentro del AAA (22.9 X 18.3 mm.).

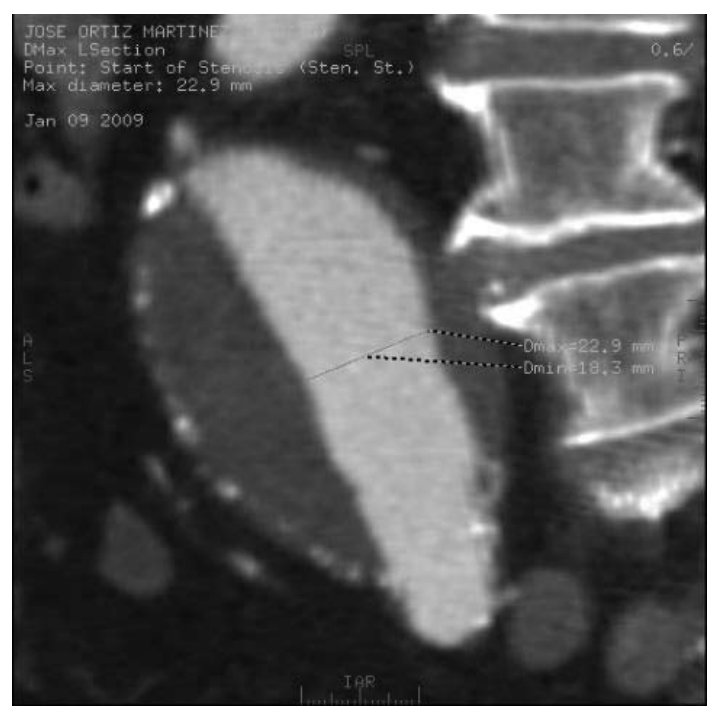

Figura 5. Tomografía contrastada de abdomen, corte sagital que muestra las dimensiones de la luz aórtica dentro del AAA (22.9 X 18.3 mm.).

\section{Discusión}

Un aneurisma aórtico abdominal se puede presentar en cualquier persona, aunque se observa con más frecuencia en hombres mayores de 60 años con uno o más factores de riesgo (hipertensión arterial sistémi$\mathrm{ca}$, enfermedad ateroesclerosa, tabaquismo, obesidad, sexo masculino, raza blanca y factores genéticos $)^{5}$. Cuanto más grande sea el aneurisma, mayor será la probabilidad de que se presente una ruptura. Cuando un aneurisma aórtico se rompe, representa una verdadera emergencia médica. Una disección aórtica ocurre cuando el revestimiento más interno de la arteria se rompe y la sangre se filtra a través de la pared arterial ${ }^{6}$.

La localización infrarrenal es la más frecuente, con afectación suprarrenal en un $5 \%$ de los casos. La presentación simultánea en otros territorios es habitual, con afectación torácica $(12 \%)$, iliaca $(25 \%)$ y periférica $(3,5 \%)$. La afectación aneurismática femoropoplítea puede acompañarse de un AAA en un $70 \%{ }^{7,8}$
La tomografía computarizada con contraste (angio-tomografía) ofrece imágenes de alta resolución y la posibilidad de una reconstrucción tridimensional. Es la exploración de elección en el diagnóstico y el estudio preoperatorio en AAA. La evaluación de la anatomía aortoiliaca, las características de la pared (inflamación, calcificación, signos de rotura y/o trombo), los vasos viscerales y las variaciones anatómicas (riñón en herradura o vena renal izquierda) se obtienen de forma detallada. Sus inconvenientes son la irradiación y el empleo de contraste 9 . La resonancia magnética (RM) con contraste presenta las mismas propiedades que la angio-tomografía, pero elimina el riesgo de nefrotoxicidad y radiaciones ionizantes. Puede sobreestimar el grado de estenosis en vasos viscerales, no detecta las calcificaciones y presenta artefactos en portadores de dispositivos de acero ${ }^{10}$

Desde su introducción por Dubost en 1951, el tratamiento de elección del AAA es la sustitución del segmento aneurismático por un injerto protésico aortoaórtico o 
bifurcado, en función de la afectación del territorio iliaco por enfermedad aterosclerótica aneurismática $\mathrm{u}$ obliterante ${ }^{11}$. Las vías de abordaje clásicas son la laparotomía media o el abordaje retroperitoneal por el flanco izquierdo. Aunque algunos autores defienden un mejor acceso por la vía retroperitoneal a la aorta suprarrenal y menor incidencia de complicaciones respiratorias.

En la historia natural de los $A A A$, el crecimiento determina la evolución hacia la rotura. Esta complicación es común en aneurismas abdominales de localización aortoiliaca y visceral, a diferencia de los que afectan al territorio periférico, en los que predomina la clínica isquémica secundaria a trombosis y/o embolización. La tasa de mortalidad en pacientes pacientes con AAA rotos es reportada entre 32 y $80 \%$. Hay una mortalidad de más del $92 \%$ en pacientes mayores de 80 años ${ }^{12}$.

Las principales complicaciones del tratamiento convencional en Ios AAA son cardiológicas $(15 \%)$, como el infarto de miocardio $(2-8 \%)$; respiratorias $(8-12 \%)$,

\section{Bibliografía}

1. Noel AA, Gloviczki P, Cherry KJ. Ruptured abdominal aortic aneurysms: the excessive mortality rate of conventional repair. J Vasc Surg. 2001;34:41-46.

2. Hirsch AT, Haskal ZJ, Hertzer NR. ACC/AHA 2005 practice guidelines for the management of patients with peripheral arterial disease (lower extremity, renal, mesenteric, and abdominal aortic). Circulation. 2006;113:463-654

3. Salhab M, Farmer J, Osman I. Impact of delay on survival in patients with ruptured abdominal aortic aneurysm. Vascular. 2006;14:38-42.

4. Sakalihasan N, Limet R, Defawe OD. Abdominal aortic aneurysm. Lancet. 2005;365:1577-89.

5. Lederle FA, Kane RL, MacDonald R, Wilt TJ. Systematic review: repair of unruptured abdominal aortic aneurysm. Ann Intern Med. 2007;146:735-741.

6. Isselbacher EM. Diseases of the aorta. In: Libby $P$, Bonow RO, mann DL, Zipes DP. Braunwald's Heart Disease: A Textbook of Cardiovascular Medicine. 8th ed. Philadelphia, Pa: Saunders Elsevier; 2007: chap 56.

7. Adamson J, Powell JT, Greenhald RM. Selection for screening for familiar aortic aneurysms. Br J Surg. 1992;79:897-8. como la neumonía $(5 \%)$; la insuficiencia renal $(5-12 \%)$, con hemodiálisis (1-6\%); la trombosis venosa $(8 \%)$; la infección de la herida quirúrgica $(<5 \%)$; el sangrado posoperatorio $(2-5 \%)$; la isquemia de las extremidades (1-4\%), y; con menos frecuencia, el accidente cerebrovascular (ACV), la lesión de la vía urinaria, la isquemia colónica izquierda, la isquemia medular y la trombosis y las infecciones protésicas ${ }^{13}$.

Durante el postoperatorio es fundamental una monitorización adecuada para mantener una hemodinamia estable. Es frecuente que además de la reposición de volumen sea necesario el uso de inotrópicos, lo que hace aconsejable el uso de un catéter en la arteria pulmonar para un mejor cálculo del débito cardíaco y presiones de llenado y su correcto manejo. Por lo general, si no ocurren las complicaciones descritas, el paciente puede ser desconectado de ventilación mecánica en un plazo de 24 - 48 horas, continuando con un postoperatorio que se asemeja a cualquier cirugía mayor.

8. Arko FR, Filis KA, Heikkinen MA. Duplex scanning after endovascular aneurysm repair: an alternative to computed tomography. Semin Vasc Surg. 2004;17:161-5.

9. Beebe HG, Kritpracha B. Computed tomography scanning for endograft planning: evolving toward three-dimensional, single source imaging. Semin Vasc Surg. 2004;17:126-34

10. Neschis DG, Fairman RM. Magnetic resonance imaging for planning aortic endograft procedures. Semin Vasc Surg. 2004;17:135-43.

11. Sicard GA, Reilly JM, Rubin BG. Transabdominal versus retroperitoneal incision for abdominal aortic surgery: report of a prospective randomized trial. J Vasc Surg. 1995;21:174-81.

12. Steyerberg EW, Kievit J, De Mol Van Otterloo JC. Perioperative mortality of elective abdominal aortic aneurysm surgery. A clinical prediction rule based on literature and individual patient data. Arch Intern Med. 1995;155:1998-2004.

13. Rockman C. Reducing complications by better case selection: Anatomic considerations. Seminars in Vascular Surgery. 2004;17:298-306. 